\title{
Estudio comparativo de la ultrasonografía abdominal en felinos menores sudamericanos en cautiverio
}

\author{
Comparative study of abdominal ultrasonography in South American \\ small feline in captivity \\ Ricardo Grandez R. ${ }^{1,2,4}$, Celeste Romero Z. ${ }^{1}$, Karina Muñoz D. ${ }^{3}$, \\ Catalina Hermoza $\mathbf{G}^{3}$
}

\section{Resumen}

\begin{abstract}
Se describió la topografía, morfología, ecotextura, ecogenicidad y arquitectura de los órganos abdominales de cuatro especies de felinos silvestres nativos del Perú mediante ultrasonografía para generar parámetros ecográficos en los felinos menores y obtener medidas comparativas entre estas especies. Los felinos se encontraban en un zoológico de Lima, Perú: dos ocelotes (Leopardus pardalis), dos oncillas (L. tigrinus), tres margay (L. wiedii) y cuatro gatos de pajonal (L. colocolo). Se utilizó un equipo de ultrasonido Esaote modelo MyLab One Vet con transductor convexo SC3123. Las características de los órganos abdominales de las especies estudiadas fueron similares en ubicación, forma, ecogenicidad y ecotextura a los descritas previamente en margay y felinos domésticos. Las dimensiones de los órganos presentaron una variación de acuerdo con el tamaño de cada especie; siendo mayores en el ocelote, seguido por el margay, el gato de pajonal, y finalmente la oncilla.
\end{abstract}

Palabras clave: ultrasonografía, abdominal, felino, silvestre

${ }^{1}$ Sección de Biociencias y Ciencias Clínicas, Departamento Académico de Medicina Veterinaria y Zootecnia, Facultad de Medicina Veterinaria y Zootecnia, Universidad Peruana Cayetano Heredia, Lima, Perú

${ }^{2}$ Laboratorio de Vida Silvestre, Facultad de Medicina Veterinaria y Zootecnia, Universidad Peruana Cayetano Heredia, Lima, Perú

${ }^{3}$ Patronato Parque de las Leyendas

${ }^{4}$ E-mail: rgrandez@hotmail.com

Recibido: 29 de julio de 2020

Aceptado para publicación: 7 de enero de 2021

Publicado: 24 de abril de 2021 
The topography, morphology, ecotexture, echogenicity and architecture of the abdominal organs of four species of wild feline native to Peru were described through ultrasonography to generate ultrasound parameters in the smaller felines and obtain comparative measurements between these species. The felines were in a zoo in Lima, Peru: two ocelots (Leopardus pardalis), two oncillas (L. tigrinus), three margay (L. wiedii) and four Pampas cats (L. colocolo). An Esaote ultrasound equipment, model MyLab One Vet with convex transducer SC3123 was used. The characteristics of the abdominal organs of the studied species were similar in location, shape, echogenicity and ecotexture to those previously described in margay and domestic cats. The dimensions of the organs presented a variation according to the size of each species; being largest in the ocelot, followed by the margay, the Pampas cat, and finally the oncilla.

Key words: ultrasonography, abdominal, feline, wild

\section{INTRODUCCIÓN}

La familia Felidae consta de tres géneros sudamericanos: Puma, Panthera y Leopardus. En este último se encuentran los felinos menores o pequeños, con 10 especies, de las cuales solo seis habitan en Perú, siendo L. colocolo, L. jacobitus, L. pajeros, $L$. pardalis, L. tigrinus y L. wiedii (Clavijo y Ramírez, 2009). Se les encuentra entre la Cordillera de los Andes hasta las sabanas húmedas del pantanal de Brasil (Lucherini et al., 2005); sin embargo, las presiones, tanto humanas como naturales, están marcando su desaparición paulatina debido a la caza furtiva, el comercio y la destrucción de su hábitat (Pacheco, 2011). De estos, solo $L$. tigrinus se encuentra catalogado como vulnerable en la lista roja de la Unión Internacional para la Conservación de la Naturaleza (UICN, 2016)

Estas y otras especies de felinos menores pueden encontrarse en zoológicos nacionales (PATPAL, 2017), donde se realizan programas de bienestar animal que incluyen enriquecimiento ambiental, manejo alimenticio y programas de control sanitario. Este último incluye exámenes físicos y pruebas diagnós- ticas y, en caso de que se considere necesario, se realizan análisis hematológicos, bioquímicos o imagenológicos (Mellor et al., 2015).

La ecografía es un método diagnóstico no invasivo, rápido y de fácil uso, utilizada especialmente para el estudio de tejidos blandos, y permite reconocer masas parenquimatosas y estructuras cavitarias (Gonzales, 2012). Mediante esta técnica se puede determinar la acumulación de líquidos en las cavidades, como la peritoneal y pleural (González, 2012). Asimismo, en el caso de animales de compañía se pueden observar patologías como piometra y quistes foliculares en hembras e hiperplasia prostática y patologías testiculares en machos (Giraldo, 2012; Matton y Nyland, 2015a,b).

Existen pocos estudios referidos al uso de la ecografía en felinos pequeños. Jarreta et al. (2004) realizaron el estudio ultrasonográfico del riñón de la oncilla (L. tigrinus); Carvalho et al. (2007) evaluaron los parámetros ecocardiográficos en la misma especie; en tanto que Grandez et al. (2019) describieron las características ecográficas de los órganos abdominales en el margay ( $L$. wiedii). 

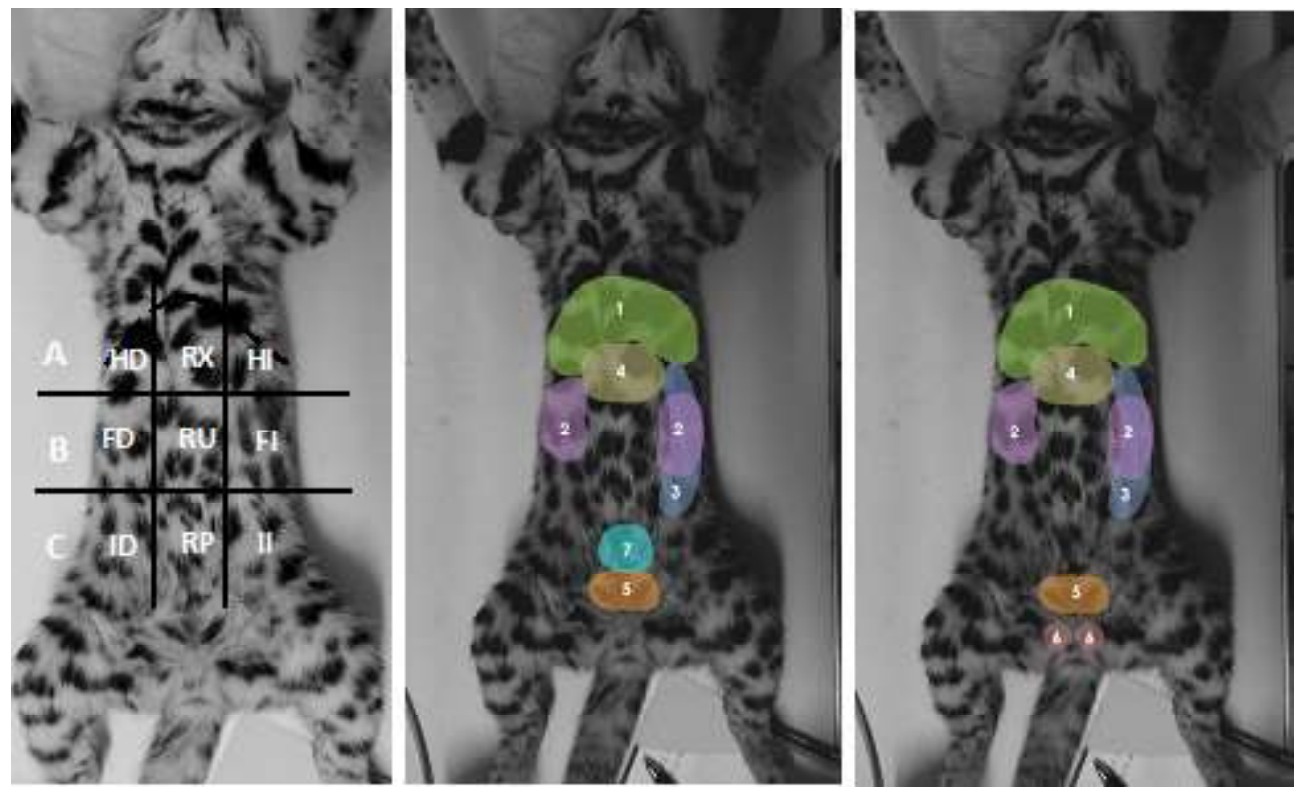

Figura 1. Regiones y órganos del abdomen de felinos menores, ocelote (Leopardus pardalis), margay (L. wiedii), gato de pajonal (L. colocolo) y oncilla (L. tigrinus) del zoológico Parque de las Leyendas (Lima, Perú). Imagen izquierda; A: Abdomen craneal (epigastrio); B: Abdomen medio (mesogastrio); C: Abdomen caudal (hipogastrio). HD: Hipocondrio derecho; HI: Hipocondrio izquierdo; RX: Región xifoidea; FD: Flanco derecho; FI: Flanco izquierdo; RU: Región umbilical; ID: Inguinal derecho; II: Inguinal izquierdo; RP: Región púbica. Imagen central: Espécimen hembra. Imagen derecha: espécimen macho. 1: Hígado; 2: Riñones; 3: Bazo; 4: Estómago; 5: Vejiga; 6: Testículos; 7: Útero

El presente trabajo tuvo como finalidad describir la topografía, morfología, ecotextura, ecogenicidad y arquitectura de los órganos abdominales de felinos del género Leopardus mediante ultrasonografía, para generar parámetros ecográficos en estas especies. La información generada permitirá al Médico Veterinario dedicado a la medicina de fauna silvestre identificar alteraciones o patologías, así como establecer el protocolo terapéutico, quirúrgico o medidas preventivas para asegurar la salud y calidad de vida de estas especies, contribuyendo de esta manera a su conservación.

\section{Materiales y Métodos}

El estudio se llevó a cabo en las instalaciones del Hospital Veterinario del zoológico del Patronato del Parque de las Leyendas (PATPAL), ubicado en el distrito de San Miguel, Lima (Perú). El tamaño de muestra correspondió al total de ejemplares de felinos menores sudamericanos mantenidos en cautiverio en PATPAL. El examen ultrasonográfico fue realizado como parte del control sanitario anual programado para los felinos menores. 
Ninguno de los animales evaluados por el médico veterinario presentó signos de enfermedad, ni su historial médico registró enfermedad los últimos tres meses; sin embargo, dos oncillas fueron excluidas del trabajo por su avanzada edad (18 y 20 años) y el riesgo que conllevaría la anestesia general para realizar el procedimiento. Los animales considerados en el estudio correspondieron a dos ocelotes ( $L$. pardalis), dos oncillas ( $L$. tigrinus), tres margay (L. wiedii) y cuatro gatos de pajonal (L. colocolo). Todos los especímenes provinieron de decomisos por tenencia o tráfico ilegal y su alimentación consistía en carne cruda y suplementación de calcio.

Los resultados de los análisis clínicos realizados no mostraron anormalidades en los animales, tanto en la evaluación clínica previa y posterior a la anestesia, ni en la reevaluación dos meses después de realizar el procedimiento.

Los animales fueron anestesiados, con base al peso histórico previo, siguiendo el protocolo de contención química: ketamina 5-10 $\mathrm{mg} / \mathrm{kg}$, xilazina $1 \mathrm{mg} / \mathrm{kg}$ y midazolam $0.1-0.3$ $\mathrm{mg} / \mathrm{kg}$ por vía intramuscular (Carpenter, 2001). Los animales fueron pesados y se les tomó una muestra de sangre de la vena inguinal para análisis de laboratorio de control correspondiente.

Para la ejecución de las ecografías, los animales se colocaron en posición ventrodorsal y lateral, tanto izquierda como derecha. El pelaje fue humedecido con alcohol y se aplicó abundante gel ecográfico para obtener una mejor imagen ecográfica, debido a que los animales no fueron rasurados. Se empleó el equipo ecográfico ESAOTE, modelo MyLab ${ }^{\mathrm{TM}}$ One Vet, con el transductor microconvexo SC3123, 4-9 MHz. Las imágenes y videos fueron almacenadas en formato digital.
Con fines prácticos y para una mejor descripción de la topografía de los órganos abdominales, se emplearon líneas imaginarias que dividieron el abdomen en abdomen craneal (epigastrio) que comprendió el hipocondrio derecho e izquierdo y la región xifoidea, el abdomen medio (mesogastrio) que comprendió el flanco derecho e izquierdo y la región umbilical y, por último, el abdomen caudal (hipogastrio) que incluyó la región inguinal derecha e izquierda y la región púbica (adaptado de Howard y De Lahunta, 1991). Además, se elaboró un esquema de la silueta del felino y de la topografía de los órganos abdominales evaluados (Figura 1).

El examen ecográfico se inició visualizando vejiga, útero y próstata en abdomen caudal; luego se visualizó bazo, riñones $\mathrm{y}$ asas intestinales en abdomen medio; $\mathrm{y}$ finalmente se visualizó hígado, vesícula biliar y estómago en abdomen craneal. Durante el examen se evaluaron las características ecográficas y dimensiones de todos los órganos observados.

Se empleó estadística descriptiva para comparar los datos de las dimensiones de los órganos evaluados, empleando la media como medida de tendencia central y la desviación estándar como medida de dispersión.

\section{Resultados}

De las cuatro especies estudiadas, el ocelote (L. pardalis) tuvo el mayor peso promedio ( $15.0 \mathrm{~kg}$; 1 hembra y 1 macho), seguido por el margay (L. wiedii) con $4.37 \mathrm{~kg}$ (2 hembras y 1 macho), el gato de pajonal ( $L$. colocolo) con $3.33 \mathrm{~kg}$ ( 3 hembras y $1 \mathrm{ma}-$ cho) y la oncilla (L. tigrinus) con $2.12 \mathrm{~kg}$ (1 hembra y 1 macho). Los 11 animales eran adultos.

Las características ecográficas (topografía, ecotextura, ecogenicidad, arquitectura de cada órgano) se presentan en los cua- 
Cuadro 1. Características ecográficas: topografía, forma, márgenes, ecotextura, ecogenicidad y arquitectura de órganos abdominales en 11 felinos menores: ocelote (L. pardalis), margay (L. wiedii), gato de pajonal $(L$. colocolo) y oncilla (L. tigrinus). Zoológico Parque de las Leyendas, LimaPerú (Parte I)

\begin{tabular}{|c|c|c|c|c|}
\hline & Vejiga & Riñones & Hígado & V. biliar \\
\hline Topografía & $\begin{array}{l}\text { Hipogastrio } \\
\text { región púbica }\end{array}$ & $\begin{array}{l}\text { Mesogastrio, } \\
\text { flancos izquierdo y } \\
\text { derecho; este último } \\
\text { ligeramente más } \\
\text { craneal }\end{array}$ & Epigastrio & $\begin{array}{l}\text { Epigastrio, entre } \\
\text { región xifoidea } \\
\text { e hipocondrio } \\
\text { derecho }\end{array}$ \\
\hline $\begin{array}{l}\text { Forma y } \\
\text { márgenes }\end{array}$ & $\begin{array}{l}\text { Sacular } \\
\text { redondeada } \\
\text { con márgenes } \\
\text { definidos }\end{array}$ & $\begin{array}{l}\text { Ovalado, con } \\
\text { márgenes } \\
\text { redondeados y } \\
\text { bordes definidos }\end{array}$ & $\begin{array}{l}\text { Compacto, de } \\
\text { bordes } \\
\text { continuos }\end{array}$ & $\begin{array}{l}\text { Piriforme o } \\
\text { seudo lobulada, } \\
\text { de paredes } \\
\text { delgadas y finas }\end{array}$ \\
\hline $\begin{array}{l}\text { Ecotextura } \\
\text { ecogenicidad y } \\
\text { arquitectura }\end{array}$ & $\begin{array}{l}\text { Contenido } \\
\text { anecoico y } \\
\text { paredes } \\
\text { delgadas, } \\
\text { ecogenicidad } \\
\text { media }\end{array}$ & $\begin{array}{l}\text { Corteza renal } \\
\text { ecogénica, de } \\
\text { granularidad media, } \\
\text { médula anecoica y } \\
\text { pelvis renal } \\
\text { hiperecogénica }\end{array}$ & $\begin{array}{l}\text { Parénquima } \\
\text { ecogénico } \\
\text { ecotextura } \\
\text { granular fina a } \\
\text { media }\end{array}$ & $\begin{array}{l}\text { Contenido } \\
\text { anecoico, pared } \\
\text { de discreta } \\
\text { ecogenicidad }\end{array}$ \\
\hline
\end{tabular}

dros 1 y 2. No se observaron diferencias resaltantes entre las especies estudiadas.

Las dimensiones de cada órgano por especie se presentan en el Cuadro 3. Se pudo obtener las medidas de casi la totalidad de los órganos de los individuos muestreados, a excepción de la medición de la vejiga de un gato de pajonal hembra que no tenía contenido, y los testículos de un ocelote macho castrado. Además, tampoco se llegaron a observar los cuernos uterinos y ovarios, a excepción de un ocelote hembra. Del análisis de las dimensiones de los órganos abdominales se puede inferir que las dimensiones fueron relativamente mayores en órganos de especies de mayor tamaño.
La vejiga urinaria se ubicó en el hipogastrio. Posee una forma sacular redondeada con márgenes regulares y definidos, su tamaño varió dependiendo del grado de distensión. Su pared fue delgada y ecogénica con capas poco diferenciadas (Figura 2A). No se observaron diferencias en el grosor de la pared de la vejiga entre especies (Cuadro 3).

Los riñones se ubicaron en las regiones del flanco izquierdo y derecho, siendo el último de ubicación más craneal. De forma ovalada, bordes definidos y capsula ecogénica. La corteza renal se pudo apreciar hiperecogénica con respecto al hígado e iso o hipo ecogénico con respecto al bazo (Figura 2B). La medula renal se apreció anecoica y la 
Cuadro 2. Características ecográficas: topografía, forma, márgenes, ecotextura, ecogenicidad y arquitectura de órganos abdominales en 11 felinos menores: ocelote (L. pardalis), margay (L. wiedii), gato de pajonal ( $L$. colocolo) y oncilla (L. tigrinus). Zoológico Parque de las Leyendas, LimaPerú (Parte II)

\begin{tabular}{|c|c|c|c|c|}
\hline & Estómago & Bazo & Útero & Testículos \\
\hline Topografía & $\begin{array}{l}\text { Límite de } \\
\text { epigastrio y } \\
\text { mesogastrio, } \\
\text { caudal al hígado }\end{array}$ & $\begin{array}{l}\text { Mesogastrio, } \\
\text { flanco izquierdo, } \\
\text { entre estómago y } \\
\text { riñón }\end{array}$ & $\begin{array}{l}\text { Hipogastrio, } \\
\text { región púbica } \\
\text { entre vejiga y } \\
\text { colon }\end{array}$ & $\begin{array}{l}\text { Región } \\
\text { perineal, en las } \\
\text { bolsas } \\
\text { escrotales }\end{array}$ \\
\hline $\begin{array}{l}\text { Forma y } \\
\text { márgenes }\end{array}$ & $\begin{array}{l}\text { Sacular e } \\
\text { irregular de } \\
\text { márgenes } \\
\text { continuos }\end{array}$ & $\begin{array}{l}\text { Alargado, } \\
\text { cabeza más } \\
\text { ancha que cola, } \\
\text { de bordes bien } \\
\text { definidos }\end{array}$ & $\begin{array}{l}\text { Estructura } \\
\text { tubular, de } \\
\text { bordes } \\
\text { continuos }\end{array}$ & $\begin{array}{l}\text { Ovalados con } \\
\text { márgenes finos } \\
\text { bien definidos }\end{array}$ \\
\hline $\begin{array}{l}\text { Ecotextura } \\
\text { ecogenicidad y } \\
\text { arquitectura }\end{array}$ & $\begin{array}{l}\text { Presencia de } 4 \\
\text { capas de } \\
\text { ecogenici-dad } \\
\text { alternada }\end{array}$ & $\begin{array}{l}\text { Parénquima } \\
\text { homogéneo, } \\
\text { granularidad } \\
\text { densa y fina, con } \\
\text { bordes } \\
\text { hiperecogénicos }\end{array}$ & $\begin{array}{l}\text { Capas poco } \\
\text { definidas, con } \\
\text { ecogenici-dad } \\
\text { alternada }\end{array}$ & $\begin{array}{l}\text { Ecogenicidad } \\
\text { media } \\
\text { ecotextura fina } \\
\text { y homogénea, } \\
\text { se observa } \\
\text { medisatinum } \\
\text { testits }\end{array}$ \\
\hline
\end{tabular}

pelvis renal fue hiperecogénica. Los riñones presentaron diferencia en tamaño según las especies, presentando mayores dimensiones las especies de mayor tamaño, evidenciando proporcionalidad (Cuadro 3). No se encontraron diferencias por ecogenicidad, morfología o topografía entre especies.

El bazo se ubicó entre el estómago y polo caudal del riñón izquierdo proyectándose hacia caudal. En la región del flanco izquierdo presentó bordes hiperecogénicos bien definidos. Presentó forma alargada, el parénquima fue homogéneo de granularidad densa y fina, y la ecogenicidad fue mayor o igual a la corteza renal y mayor al parénquima hepático (Figura 3A). El bazo presentó, al igual que los riñones, diferencia en tamaño según la especie, mostrando proporcionalidad al tamaño de la especie (Cuadro 3). No se encontraron diferencias por ecogenicidad, morfología o topografía entre especies.

El hígado se ubicó en la región xifoidea, hipocondrio derecho e izquierdo, limitando cranealmente con el diafragma. Presentó parénquima compacto, hipoecogénico con relación al bazo y al riñón, de bordes continuos y ecotextura homogénea de granularidad fina a media; pudiendo apreciarse tractos anecoicos que correspondieron a venas hepáticas y portales. En este órgano se pudo evaluar la ecogenicidad, topografía y morfología sin encontrar variación entre las especies. 
Cuadro 3. Dimensiones ecográficas $(\mathrm{cm})$ de los órganos abdominales no reproductivos de cuatro especies de felinos menores (L. pardalis, $L$. colocolo, L. tigrinus, L.wiedii) del Zoológico Parque de las Leyendas (Lima, Perú)

\begin{tabular}{|c|c|c|c|c|c|c|c|c|c|c|c|}
\hline \multirow{2}{*}{ 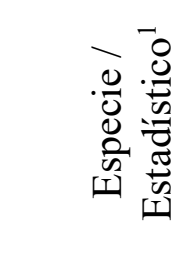 } & & \multirow[t]{2}{*}{$\begin{array}{l}\frac{000}{y} \\
0 \\
0 \\
0 \\
0\end{array}$} & \multirow[t]{2}{*}{$\frac{\pi}{000}$} & \multirow[t]{2}{*}{ 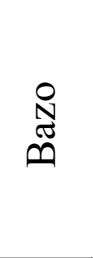 } & \multirow[t]{2}{*}{ 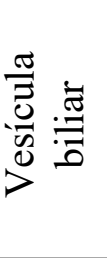 } & \multirow[t]{2}{*}{ 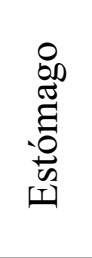 } & \multirow[t]{2}{*}{ 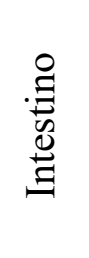 } & \multicolumn{2}{|c|}{ 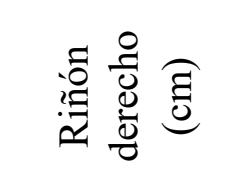 } & \multicolumn{2}{|c|}{ 记 } \\
\hline & & & & & & & & & & & \\
\hline \multirow{4}{*}{$\begin{array}{l}\text { Ocelote } \\
(\text { L. } \\
\text { pardalis }) \\
\mathrm{n}=2\end{array}$} & $\bar{v}$ & & & & & & & & & & \\
\hline & $\mathrm{DS}$ & & & & & & 04 & 76 & & & .03 \\
\hline & M & 0 & & 1.15 & & & 52 & 3 & & & 208 \\
\hline & $\mathrm{M}$ & 00 & & & 5 & & 7 & 0 & 3.31 & & 3.02 \\
\hline \multirow{4}{*}{$\begin{array}{l}\text { Margay } \\
(L . \\
\text { wiedii) } \\
\mathrm{n}=3\end{array}$} & $X$ & & & & & & & 0 & 2.16 & & 2.68 \\
\hline & DS & 60 & & y & & & 04 & 66 & 0.44 & & 1.13 \\
\hline & $\mathrm{M}$ & U & & & & & & U & & & 1.60 \\
\hline & 10 & & & & & & & & & & \\
\hline \multirow{4}{*}{$\begin{array}{l}\text { Gato de } \\
\text { pajonal } \\
\text { (L. } \\
\text { colocolo) } \\
\mathrm{n}=4\end{array}$} & & & & & & & & & & & 2.16 \\
\hline & $\mathrm{S}$ & & & & & & & & & & 0.34 \\
\hline & M & 2.50 & & 0,50 & & & 2 & 4 & 1.91 & 7 & 1.73 \\
\hline & $\mathrm{M}$ & 4.40 & 0.23 & ) & 150 & $0.4 J$ & 2 & 98 & 2 & 75 & 2.5 \\
\hline \multirow{4}{*}{$\begin{array}{l}\text { Oncilla } \\
\text { (L. } \\
\text { tigrinus) } \\
\mathrm{n}=2\end{array}$} & & & & & & & & & & & 0.93 \\
\hline & DS & 23 & & & & & 4 & 08 & 1.23 & 3 & 1.32 \\
\hline & $\mathrm{M}$ & 1.96 & & & & & .44 & 2.76 & 0.00 & 2.81 & 0.00 \\
\hline & $\mathrm{M}$ & .28 & 20 & .74 & .93 & .20 & 0.50 & 2.87 & 1.74 & 2.85 & 1.86 \\
\hline
\end{tabular}

${ }^{1} \mathrm{X}$ : media; DS: desvío estándar; m: valor mínimo; M: valor máximo

La vesícula biliar se ubicó entre los lóbulos hepáticos lateral y medial derechos, en la región xifoidea e hipocondrio derecho, insertado en el parénquima hepático. De aspecto piriforme y en la mayoría de las especies era seudo lobulada. Presenta pared delgada y fina, con contenido anecoico (Figura 3B). El tamaño de la vesícula biliar presentó proporcionalidad acorde al tamaño de la especie (Cuadro 3).
El estómago se ubicó caudal al hígado, principalmente en el límite de la región xifoidea y umbilical. Es un órgano saculado, alargado e irregular, de tamaño variable, dependiendo del contenido y distensión; sus paredes presentaban capas de ecogenicidad alternada: serosa y subserosa hiperecogénicas, muscular hipoecogénica, submucosa hiperecogénica, y mucosa hipoecogénica, siendo esta última de disposición ondulada, 

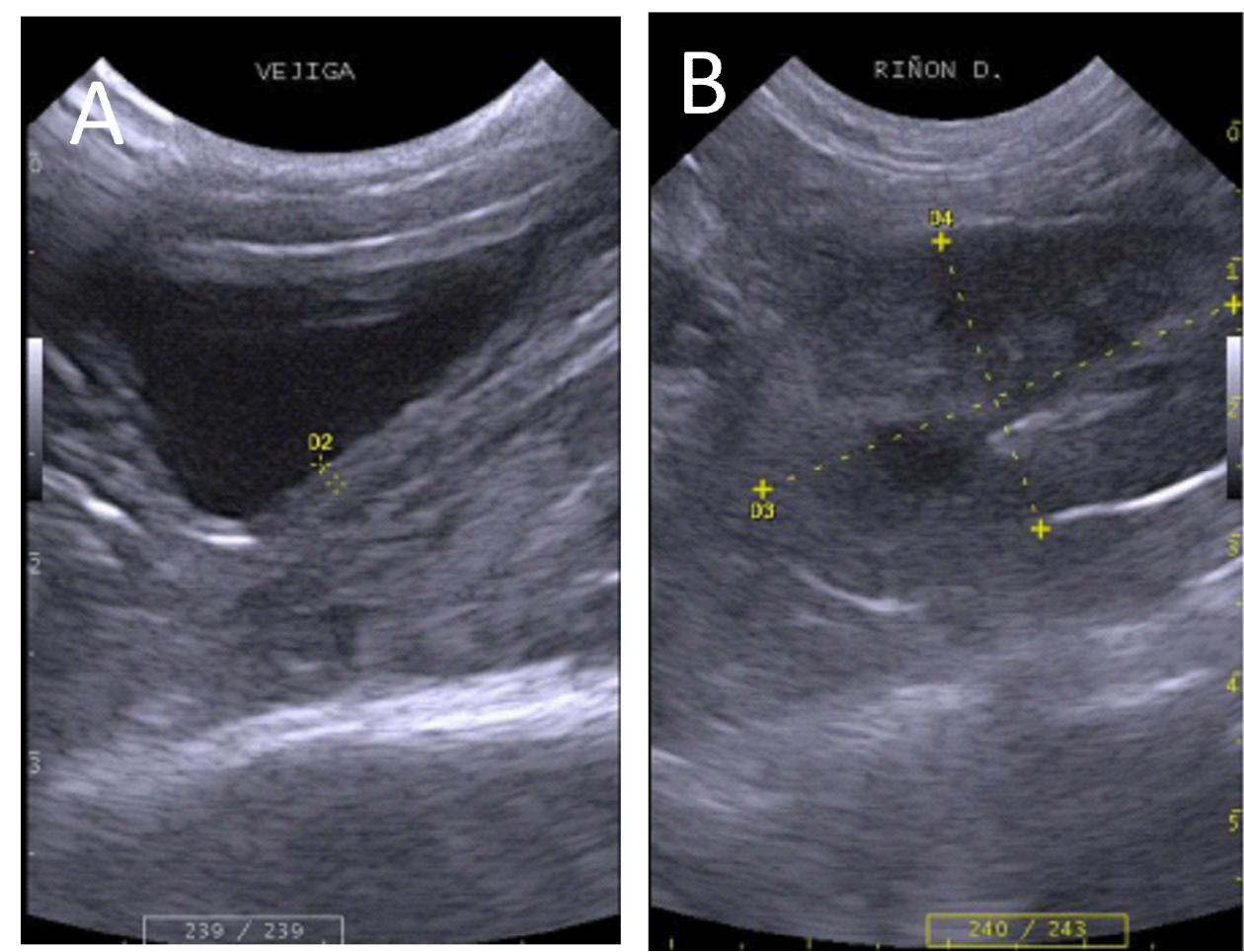

Figura 2. Imagen ultrasonográfica de vejiga y riñón. A, vejiga urinaria de gato de pajonal $(L$. colocolo) hembra ( $0.12 \mathrm{~cm}$ de espesor). $\mathrm{B}$, riñón derecho de margay (L. wiedii) hembra $(3.68 \times 2.20 \mathrm{~cm})$

por la presencia de pliegues (Figura 4A). Las asas intestinales se ubicaron en la región umbilical, con disposición de capas similares a las del estómago, sin presencia de pliegues y el lumen con patrón mucoso (Figura 4B).

El grosor de la pared del estómago y el diámetro de los intestinos varió proporcionalmente acorde al tamaño de la especie. No hubo variación en cuanto a ecogenicidad y ecotextura, ya que todas las especies presentaron las capas características del estómago e intestinos (Cuadro 3).

El cuerpo del útero se ubicó en la región púbica, entre la vejiga y colon. Se observó como una estructura tubular de bordes continuos y redondeados. Presentó una pared gruesa con capas poco definidas y lumen con patrón mucoso, de ecogenicidad intermedia (Figura 5A). Solo se pudo identificar un ovario en un ocelote con tamaño de $1.88 \mathrm{x}$ $1.33 \mathrm{~cm}$, ya que presentó un folículo ovárico (0.61 cm de diámetro); al encontrase en fase de estro (Figura 5B); no obstante, en este espécimen no se identificó el cuerpo uterino. Las dimensiones del útero presentaron proporcionalidad al tamaño entre las tres especies donde se lograron identificar, siendo en el margay de $0.89 \times 0.74 \mathrm{~cm}(\mathrm{n}=2)$, en el gato del pajonal de $0.69 \times 0.60 \mathrm{~cm}(\mathrm{n}=3)$, y en la única oncilla evaluada de $0.34 \times 0.30 \mathrm{~cm}$ de altura y ancho al corte transversal, respectivamente. No se observaron diferencias en cuanto a ecogenicidad, ecotextura y topografía.

Los testículos, órganos pares, se ubicaron en la zona perineal. Presentaron forma ovalada con una túnica albugínea gruesa e hiperecoica. El parénquima con ecogenicidad media y ecotextura fina y homogénea, con una línea central hiperecogénica que corres- 

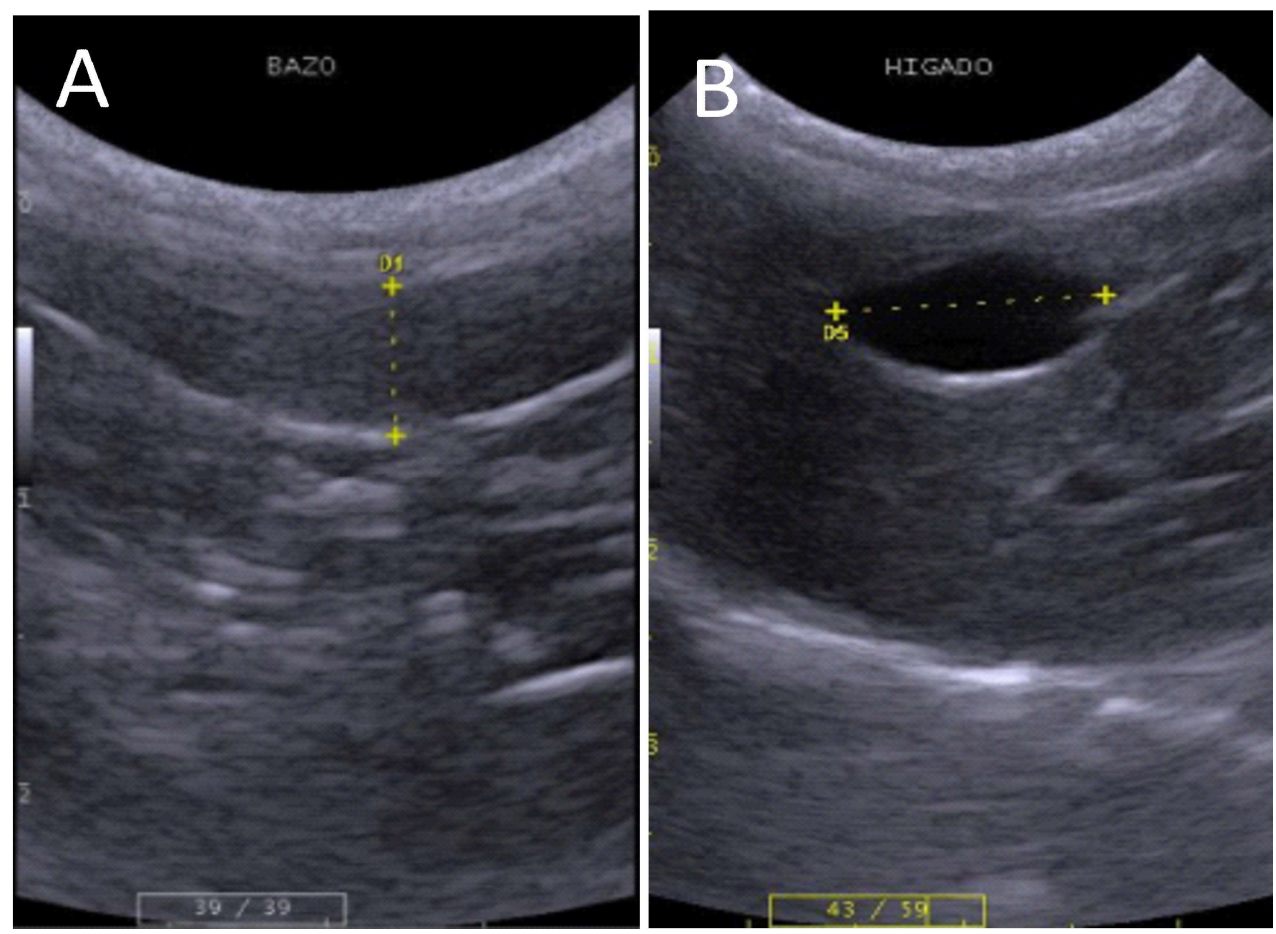

Figura 3. Imagen ultrasonográfica del bazo, hígado y vesícula biliar. A, bazo de oncilla $(L$. tigrinus) macho ( $0.51 \mathrm{~cm}$ espesor). B, hígado y vesícula biliar, oncilla hembra (1.26 cm de longitud)
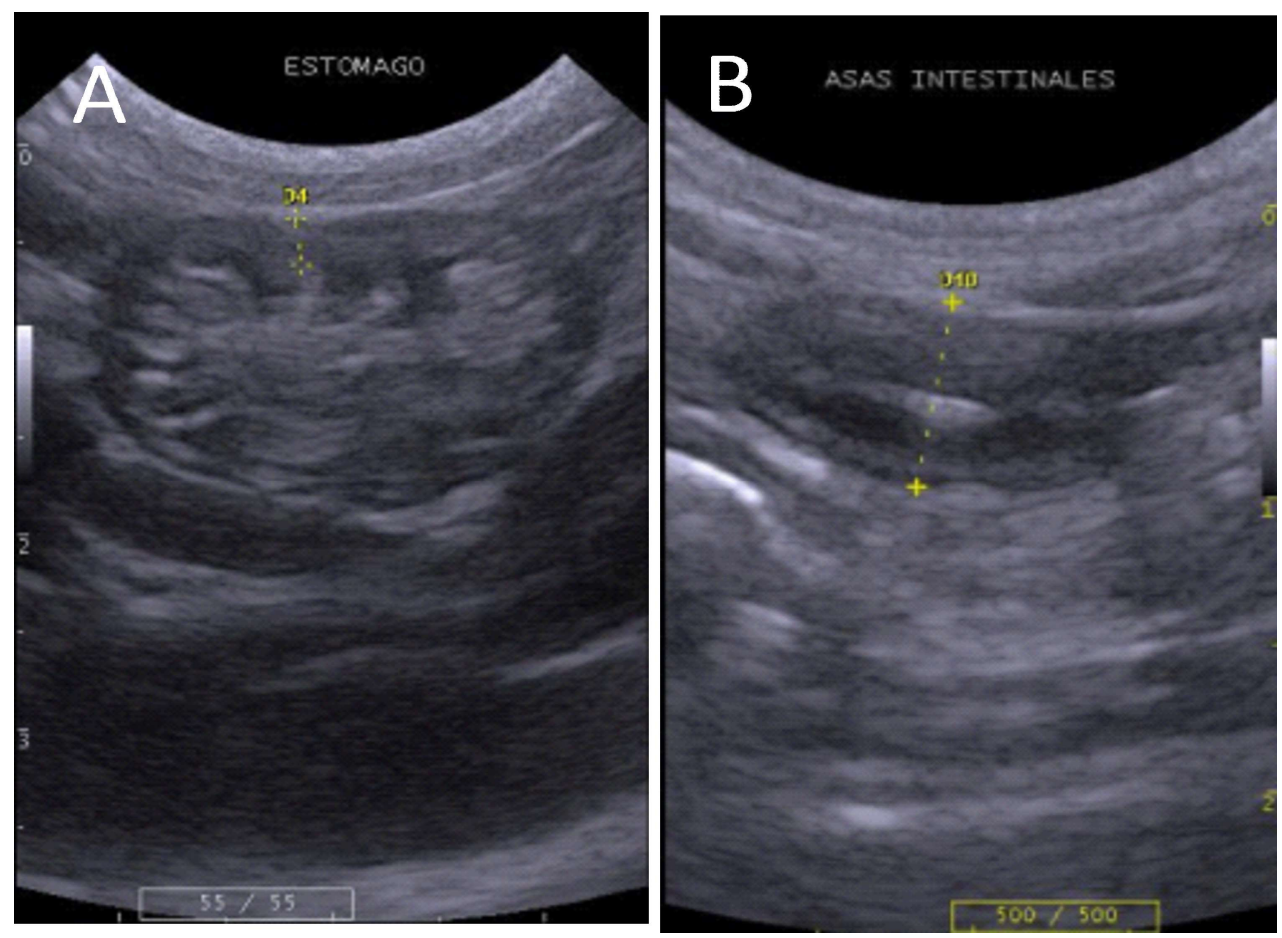

Figura 4. Imagen ultrasonográfica del estómago y asas intestinales. A, estómago con escaso contenido, gato de pajonal (L. colocolo) hembra $(0.24 \mathrm{~cm}$ de espesor). B, asas intestinales, margay ( $L$. wiedii) macho $(0.64 \mathrm{~cm}$ de diámetro) 

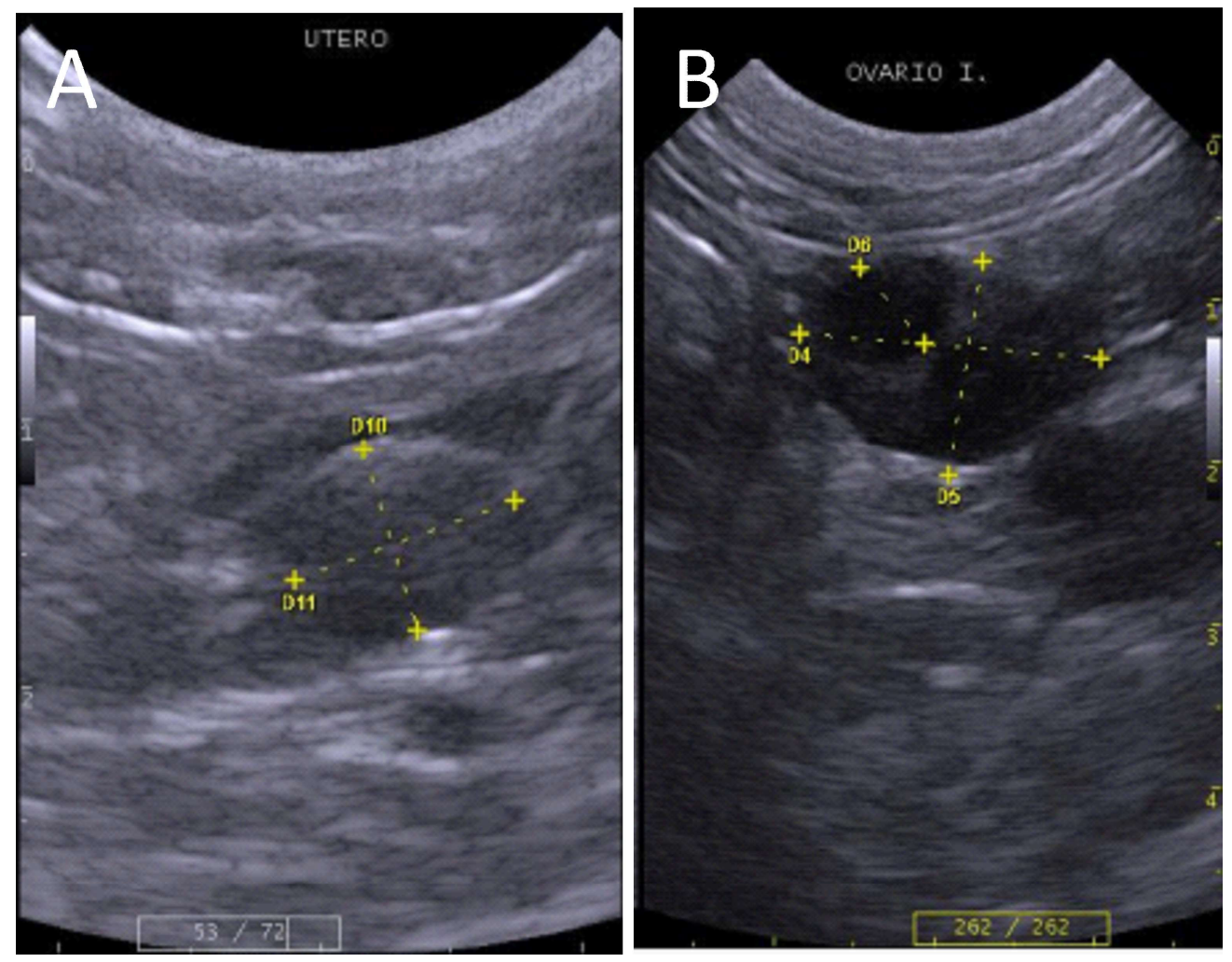

Figura 5. Imagen ultrasonográfica del cuerpo del útero y ovario. A, cuerpo del útero de gato de pajonal (L. colocolo $)(0.71 \times 0.89 \mathrm{~cm})$. B, ovario izquierdo con folículo ovárico, ocelote (L. pardalis) hembra $(1.88 \times 1.33$; folículo de $0.61 \mathrm{~cm})$

pondió al mediastinum testis (Figura 6). Las dimensiones del único par de testículos por especie fueron: Margay, testículo derecho $1.92 \times 1.16 \mathrm{~cm}$ e izquierdo $1.92 \times 1.08 \mathrm{~cm}$; Gato del pajonal, testículo derecho $1.63 \times 1.01$ $\mathrm{cm}$ e izquierdo $1.35 \times 0.97 \mathrm{~cm}$; Oncilla, testículo derecho $1.49 \times 1.09 \mathrm{~cm}$ e izquierdo 1.51 x $0.98 \mathrm{~cm}$ de largo y ancho, respectivamente.

\section{Discusión}

Con base a estudios previos realizados en margay por Grandez et al. (2019) y en oncilla Jarreta et al. (2004) se pudo comparar la información obtenida en este estudio con ejemplares de la misma especie y con especies como el gato doméstico (Felis silvestris catus), con el que comparten características, tanto anatómicas como fisiológicas.
La ubicación, forma y características ecográficas de la vejiga coincide con lo descrito para el margay por Grandez et al. (2019), y para felinos domésticos por Sutherland-Smith y Penninck (2015). En el caso de los riñones las características topográficas de ecotextura, ecogenicidad y arquitectura fueron similares a las descritas por Jarreta et al. (2004) en oncillas, y por Espada (2015) en felinos domésticos.

El bazo y el hígado presentaron características de ecotextura, ecogenicidad, forma y arquitectura similar al descrito para felinos domésticos por Domínguez (2015b), Novellas (2015a), Hecht y Mai (2015) y d'Anjou y Penninck (2015). La vesícula biliar presentó características similares a las descritas en felinos domésticos por otros autores (d'Anjou y Penninck, 2015; Matton et 

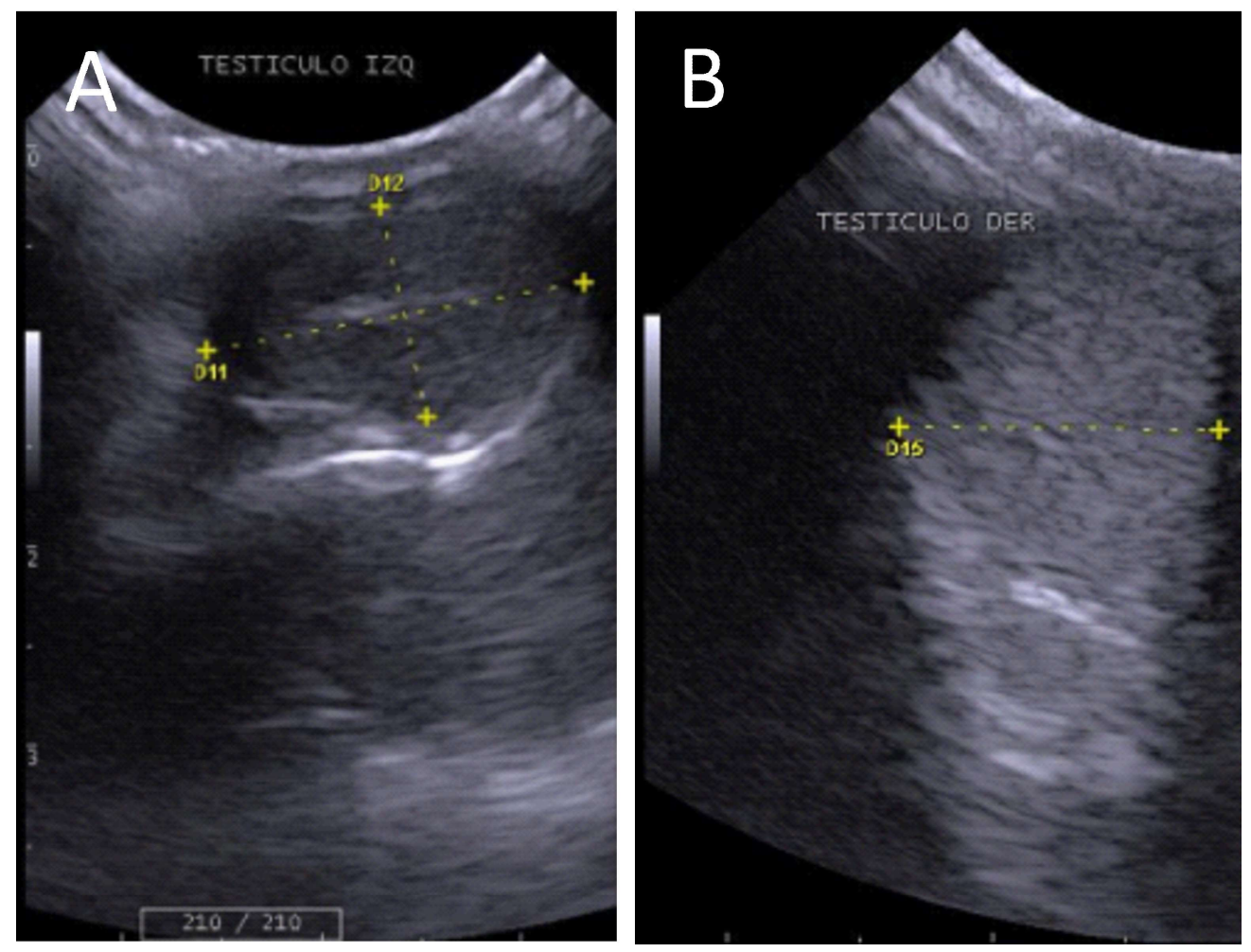

Figura 6. Imagen ultrasonográfica de los testículos. A, corte longitudinal de testículo derecho, margay (L. wiedii) $(1.92 \times 1.16 \mathrm{~cm})$ con discreta línea hiperecogénica compatible con mediastinum testis. B, corte transversal, gato de pajonal (L. colocolo) de testículo derecho $(1.21 \mathrm{~cm})$

al., 2015a; Grandez et al., 2019) en margay; asimismo, compartieron la característica de seudo o bilobulada como lo describe Novellas (2015a) en felinos domésticos. Sin embargo, se encontraron variados tamaños de la vesícula, probablemente debido al ayuno no estricto.

El estómago presentó pliegues similares a los descritos por Matton et al. (2015b) en felinos domésticos; en tanto que las asas intestinales presentaron capas similares al estómago, coincidente con la ecogenicidad descrita por Novellas (2015b) en felinos domésticos.
En el útero, tanto las características ecogénicas, ecotextura, ubicación como forma coinciden con lo descrito por Pollard y Hecht (2015) en felinos domésticos y por Grandez et al. (2019) para el margay. Los testículos se encontraron en bolsas escrotales, mostrando características similares a las descritas (Domínguez, 2015a; Matton y Nyland, 2015b; Pollard y Hecht, 2015) en el felino doméstico. El único ovario observado correspondió a un ocelote hembra en fase de estro, fase del ciclo estrual donde folículos triplican su tamaño comparado a la fase de anestro, donde son pequeños y ovalados siendo de dificilobservación (Matton y Nyland, 2015a; Pollard y Hecht, 2015) para felinos domésticos. 
Se puede concluir que las características de los órganos abdominales mantienen una distribución similar entre sí, y fueron similares en ubicación, forma, ecogenicidad, ecotextura y en relación con las características topográficas del gato doméstico descritas por Novellas et al. (2015) y en el margay descrito por Grandez et al. (2019). Asimismo, las dimensiones de los órganos presentaron una variación de proporcionalidad acorde al tamaño de la especie, siendo mayores en el ocelote, seguido por el margay, el gato de pajonal, y la oncilla.

Se observó que los órganos abdominales

\section{Agradecimiento}

Los autores agradecen al Patronato del Parque de las Leyendas por las facilidades brindadas en la realización del presente estudio y a la empresa Ultrason Vet EIRL por facilitar el equipo ecográfico empleado.

\section{Literatura Citada}

1. Carpenter JW. 2001. Exotic animal formulary. $3^{\text {rd }}$ ed. Missouri: Elsevier Saunders. 496 p.

2. Carvalho P, Pereira G, Petrus L, Soares E, Michima L, Larsson M. 2007. Avaliação de alguns parâmetros ecocardiográficos do gato-do-mato (Leopardus tigrinus), mantido em cativeiro e submetido à anestesia com xilazina e quetamina. Arq Bras Med Vet Zoo 59: 695-699. doi 10.1590/S010209352007000300022

3. Clavijo A, Ramírez GF. 2009. Taxonomía, distribución y estado de conservación de los felinos suramericanos; revisión monográfica. Bol Cient Mus Hist Nat 13: 43-60.

4. d'Anjou M, Penninck D. 2015. Hígado. En: Penninck D, d'Anjou M (eds). Atlas of small animal ultrasonography. $2^{\text {nd }}$ ed. USA: Blackwell Publishing. $p$ 183-238.
5. Domínguez E. 2015a. Ecografía del aparato reproductor. En: Novellas R, Domínguez E, Espada Y, Martínez Y, Tobón M (eds). Diagnóstico ecográfico en el gato. España: Servet. p 199-210.

6. Domínguez E. 2015b. Ecografía del bazo. En: Novellas R, Domínguez E, Espada Y, Martínez Y, Tobón M (eds). Diagnóstico ecográfico en el gato. España: Servet. p 133-146.

7. Espada Y. 2015. Ecografía de los riñones y uréteres. En: Novellas R, Domínguez E, Espada Y, Martínez Y, Tobón M (eds). Diagnóstico ecográfico en el gato. España: Servet. p 157-182.

8. Gonzales G. 2012. La ecografía. Un método de diagnóstico rápido, seguro y no invasivo. PRISMA (6): 2-3 [Internet]. Disponible en: http://www.produccionanimal.com.ar/informacion_tecnica/ ecografia_ultrsonido/80-La_ecografia.pdf

9. Giraldo C. 2003. Principios básicos de ultrasonografía veterinaria. Rev MVZ Córdoba 8: 303-309. doi: 10.21897/ rmvz.508

10. Grandez R, Márquez H, Hermoza C, Valencia R. 2019. Caracterización ultrasonografica de los órganos abdominales del margay (Leopardus wiedii) en cautiverio. Rev Inv Vet Perú 30: 10091017. doi: 10.15381/rivep.v30i3.16609

11. Hecht S, Mai W. 2015. Bazo. En: Penninck D, d'Anjou M (eds). Atlas of small animal ultrasonography. $2^{\text {nd }}$ ed. USA: Blackwell Publishing. p 239-258.

12. Howard E, De Lahunta A. 1991. Miller Disección del perro. Pennsylvania, USA: Interamericana Mac Graw-Hill. 369 p.

13. Jarretta GB, Bombonato PP, Guimarães MA. 2004. Renal ultrasonographic evaluation in the oncilla (Leopardus tigrinus). J Zoo Wildl Med 35: 356-360. doi: 10.1638/02-021

14. Lucherini M, Ramiro E, Erize F, Pereyra J, Wiesel F, Parera A, Gomez T, et al.. 2005. Proyecto Felinos sudamericanos. Especies de felinos sudamericanos de interés para el banco de re- 
cursos genéticos. Fundación BBVA. [Internet]. Disponible en: http://www.gebir.csic.es/descargas/desc brgfel/Dossier_fel.pdf

15. Matton J, Nyland T. 2015a. Ovarios y útero. En: Matton J, Nyland T (eds). Small animal diagnostic ultrasound. $3^{\circ}$ ed. Missouri: Saunders. p 634-654.

16. Matton J, Nyland T. 2015b. Testículos y próstata. En: Matton J, Nyland T (eds). Small animal diagnostic ultrasound. $3^{\circ}$ ed. Missouri: Saunders. p 608-633.

17. Matton J, Nyland T, Moon M. 2015. Hígado. En: Matton J, Nyland T, editores. Small animal diagnostic ultrasound. $3^{\circ}$ ed. Missouri: Saunders. p 332-399.

18. Matton J, Nyland T, Neelis A. 2015. Estómago. En: Matton J, Nyland T (eds). Small animal diagnostic ultrasound. $3^{\circ} \mathrm{ed}$. Missouri: Saunders. p 468-500.

19. Mellor D, Hunt S, Gusset M. 2015. Cuidando la fauna silvestre: la estrategia mundial de zoológicos y acuarios para el bienestar animal. Asociación Mundial de Zoológicos y Acuarios (WAZA). [Internet]. Disponible en: http://www.waza.org/files/webcontent/1.publicsite/ 5.conservation/animal_welfare/ WAZA\%20Animal\%-20Welfare\%20Strategy\%202015_Spanish.pdf

20. Novellas R, Domínguez E, Espada Y, Martínez Y, Tobon M. 2015. Diagnóstico ecográfico en el gato. Zaragoza: Servet. 256 p.
21. Novellas R. 2015a. Ecografía del hígado y sistema biliar. En: Novellas R, Domínguez E, Espada Y, Martínez Y, Tobón M (eds). Diagnóstico ecográfico en el gato. España: Servet. p 67-96.

22. Novellas R. 2015b. Ecografía del tracto gastrointestinal. En: Novellas R, Domínguez E, Espada Y, Martínez Y, Tobón M (eds). Diagnóstico ecográfico en el gato. España: Servet. p 97-120.

23. Pacheco V. 2011. Estudio de especies CITES de carnívoros peruanos. Ministerio del Ambiente. [Internet]. Disponible en: http://sinia.minam.gob.pe/documentos/estudio-especies-citescarnivoros-peruanos-revision

24. [PATPAL] Patronato Parque De las Leyendas. 2017. Zoología. [Internet]. Disponible en: http://leyendas.gob.pe/ zoologia/

25. Pollard R, Hecht S. 2015. Tracto reproductivo de la hembra y macho. En: Penninck D, d'Anjou M (eds). Atlas of small animal ultrasonography. $2^{\circ}$ ed. USA: Blackwell Publishing. p 403-421.

26. Sutherland-Smith J, Penninck D. 2015. Vejiga y uretra. En: Penninck D, d'Anjou M (eds). Atlas of small animal ultrasonography. $2^{\circ}$ ed. USA: Blackwell Publishing. p 363-385.

27. [UICN] International Union for Conservation of Nature and Natural Resources.. 2016. The IUCN Red List of threatened species. [Internet]. Available in: http://www.iucnredlist.org/ details/54012637/0 